\title{
Progesterone protects ovarian cancer cells from cisplatin-induced inhibitory effects through progesterone receptor membrane component $1 / 2$ as well as AKT signaling
}

\author{
XIAOFEI ZHU ${ }^{1 *}$, YUE HAN $^{2 *}$, ZHUYUAN FANG $^{3 *}$, WENZHONG WU ${ }^{3}$, MINGDE JI $^{1}$, \\ FENGMENG TENG $^{1}$, WEI ZHU ${ }^{4}$, XUEWEN YANG $^{1}$, XUEMEI JIA $^{5}$ and CHUNBING ZHANG ${ }^{1}$ \\ Departments of ${ }^{1}$ Laboratory Medicine, ${ }^{2}$ Gynecology and ${ }^{3}$ Cardiology, Jiangsu Provincial Hospital of \\ Traditional Chinese Medicine, Nanjing, Jiangsu 210029; Departments of ${ }^{4}$ Oncology and ${ }^{5}$ Gynecology, \\ The First Affiliated Hospital of Nanjing Medical University, Nanjing, Jiangsu 210029, P.R. China
}

Received June 25, 2013; Accepted August 2, 2013

DOI: 10.3892/or.2013.2680

\begin{abstract}
Progesterone, also known as P4 (pregn-4-ene-3, 20-dione), is a C-21 steroid hormone involved in the female menstrual cycle, pregnancy (supports gestation) and embryogenesis of humans and other species. Despite the physiological effects, P4 is also effective for the treatment of numerous pathological states, such as multiple sclerosis, rheumatoid arthritis and systemic lupus erythematosus as well as cancer. Considering the hormone microenvironment of gynecological cancers, $\mathrm{P} 4$ should be particularly noted in ovarian cancer. The present study demonstrated that $\mathrm{P} 4$ protected the ovarian cancer cell line HO-8910 from cisplatin (CDDP)-induced cell cycle arrest and restored the cell migratory capability following treatment of CDDP. Mechanistically, both progesterone receptor membrane component 1 (PGRMC1) and the progesterone receptor (PGR) were decreased in the cells treated with CDDP plus $\mathrm{P} 4$, while the level of progesterone receptor membrane component 2 (PGRMC2) was significantly elevated. Reversely, in the HO-8910 cells treated with CDDP alone, levels of both PGRMC1 and PGR were increased while the level of PGRMC2 was decreased. In addition to the receptor expression profile, the PI3K/AKT signaling pathway was also involved in the action of $\mathrm{P} 4$ in the
\end{abstract}

Correspondence to: Professor Chunbing Zhang, Department of Laboratory Medicine, Jiangsu Provincial Hospital of Traditional Chinese Medicine, Nanjing, Jiangsu 210029, P.R. China E-mail:nju_tcm@163.com

Professor Xuemei Jia, Department of Gynecology, The First Affiliated Hospital of Nanjing Medical University, Nanjing, Jiangsu 210029, P.R. China

E-mail: zxfyxjy@163.com

*Contributed equally

Key words: progesterone, ovarian cancer, AKT, migration, progesterone receptor membrane component $1 / 2$
CDDP-resistant HO-8910 cells, and a chemical inhibitor for PI3K, LY294002, significantly abolished the anti-apoptotic effect of P4. Consequently, the addition of a PI3K inhibitor to CDDP-based chemotherapy may have a more beneficial application for ovarian cancer therapy.

\section{Introduction}

Ovarian cancer is associated with the highest mortality rate among women than all other gynecological cancers in the world (1). Surgery to remove as much of the tumor as possible following platinum-based chemotherapy are the standard methods used to cure the disease (1). Although these intense surgical and chemotherapeutic treatments ease the state of illness, ovarian cancer more often than not recurs with the neoplasm usually distributed throughout the peritoneum. Again, platinum-based chemotherapy is often used to treat recurrent ovarian cancers, yet, many the ovarian cancer cells are resistant to platinum-based agents. Multiple theories have attempted to explain why cancer cells are resistant to platinum-based chemotherapy. In the case of ovarian cancer, dysfunction of sex steroids such as progesterone (P4) may play an important role. Importantly, serum P4 levels are elevated in patients with ovarian cancer when compared with age-matched controls (2), and P4 levels are also higher in the peritoneal fluid of ovarian cancer patients (3). Epidemiologically, postmenopausal smokers with significantly elevated levels of P4 show a negative prognostic effect for ovarian cancer-specific survival, while the negative effect of smoking was found to diminish with increasing time since the point a former smoker stops smoking (4-6). Although the binding of P4 to the progesterone receptor (PGR) leads to a protective effect in vitro, the antiapoptotic effect of P4 may be mediated by other less known receptors such as progesterone receptor membrane component 1 or 2 (PGRMC1/2). Research has demonstrated that P4 shows a significant anti-apoptotic effect through PGRMC1/2 in vitro and in vivo (7). These biphasic abilities of $\mathrm{P} 4$ to either induce or inhibit apoptosis could also explain why clinical trials have failed to show a beneficial effect of $\mathrm{P} 4$ on ovarian cancer progression. Regarding the ambiguous and crucial 
role of P4 in ovarian cancer, particularly in chemotherapy resistance, additional detailed mechanisms involved including PGRMC1/2 and signal pathways were thoroughly investigated in the present study.

\section{Materials and methods}

Cells and reagents. The ovarian cancer cell line HO-8910 (a human ovarian cancer cell line established from a patient with poorly-differentiated serous carcinoma) was obtained from the American Type Culture Collection (ATCC, Rockville, MD, USA). HO-8910 cells were sustained in Dulbecco's modified Eagle's medium (DMEM) (Invitrogen) with $10 \%$ (v/v) fetal bovine serum (FBS; Gibco), $100 \mathrm{IU} / \mathrm{ml}$ penicillin and $100 \mathrm{ng} /$ $\mathrm{ml}$ streptomycin (PAA Laboratories $\mathrm{GmbH}$, Pasching, Austria) in a $37^{\circ} \mathrm{C}, 5 \% \mathrm{CO}_{2}$ humidified atmosphere. Cisplatin (CDDP) was a kind gift from Wei Zhu (Department of Oncology, The First Affiliated Hospital of Nanjing Medical University, China). Progesterone (P4) and the specific inhibitor of PI3K, LY294002, were purchased from Sigma (St. Louis, MO, USA).

MTT cell viability assay. Cell viability of HO-8910 cells was examined by MTT assays according to the standard methods. Briefly, HO-8910 cells were seeded onto a 96-well plate $(2,000$ cells/well). After $12 \mathrm{~h}$, medium was replaced with conditional medium containing 10\% FBS as well as DMSO, P4 (1 $\mu \mathrm{M})$, CDDP (30 nM), or P4 $(1 \mu \mathrm{M})$ plus CDDP (30 nM), respectively. After incubation for another $48 \mathrm{~h}, 15 \mu \mathrm{l} \mathrm{MTT}(1 \mu \mathrm{g} / \mu \mathrm{l})$ was added into the medium. DMSO (200 $\mu \mathrm{l} /$ well) was used to dissolve the formazan product. Optical density was detected at a wavelength of $560 \mathrm{~nm}$.

Cell cycle analysis. The cell cycle was analyzed by flow cytometry (Beckman Coulter, Inc., Miami, FL, USA) according to the standard methods and as previously described (8).

Wound healing assay. A monolayer of HO-8910 cells at $80 \%$ confluence supplied with DMEM containing 0.5\% FBS in 12 -well plates was scratched using a sterilized $10-\mu 1$ pipette tip, washed and then subjected to P4 $(1 \mu \mathrm{M})$, CDDP $(30 \mathrm{nM})$, or P4 $(1 \mu \mathrm{M})$ plus CDDP (30 $\mathrm{nM})$, respectively. The migration of cells into the wound was imaged and monitored at 0 , 12 and $24 \mathrm{~h}$ after treatment. The images were analyzed by Image-Pro Plus software (Media Cybernetics, Silver Spring, MD, USA). Results were obtained from three independent experiments.

Transwell chamber migration assay. Migration of HO-8910 cells through $8-\mu \mathrm{m}$ pores was examined using a Transwell cell culture chamber (Corning Costar). The lower chamber was filled with DMEM containing 10\% FBS. Cells (100 $\mu \mathrm{l})$ at a density of $2 \times 10^{5} / \mathrm{ml}$ were seeded onto the upper chamber with the culture containing $\mathrm{P} 4(1 \mu \mathrm{M}), \mathrm{CDDP}(30 \mathrm{nM})$ or $\mathrm{P} 4(1 \mu \mathrm{M})$ plus CDDP (30 nM), respectively. Chambers were incubated for $18 \mathrm{~h}$ at $37^{\circ} \mathrm{C}$. The cells remaining on the top surface of the membrane were removed with application of a cotton swab followed by three PBS washes. The cells on the bottom surface of the membrane were fixed, stained $(0.1 \%$ crystal violet), and quantified by counting 5 fields on the membrane under a x20 objective.
$R T-P C R$. RT-PCR was used to characterize the expression of PGR, PGRMC1 and PGRMC2 in HO-8910 cells. Using this protocol, total RNA was isolated from HO-8910 cells using the RNeasy Plus Mini kit (Qiagen, Valencia, CA, USA). To ensure no DNA contamination of the RNA, which could lead to false-positive results, the RNA samples were treated with DNase I (Invitrogen) before reverse transcription. Then cDNA was synthesized by incubating $1 \mu \mathrm{g}$ of RNA with oligo-dT and Muloney murine leukemia virus reverse transcriptase (Invitrogen). Primers for the subsequent PCRs were as follows: PGR forward, 5'-GAT GCT ATA TTT TGC GCC TGA-3' and reverse, 5'-CTC CTT TTT GCC TCA AAC CA-3' (266 bp); PGRMC1 forward, 5'-GCA AGC TTT GGC GAA AAT CA-3' and reverse, 5'-CCC CTC GCA TGT CCA ATC AT-3' (121 bp); PGRMC2 forward, 5'-AGG GGA AGA ACC GTC AGA AT-3' and reverse, 5'-AAG CCC CAC CAG ACA TTA CA-3' (280 bp). Reaction times were: $1 \mathrm{~min}$ at $94^{\circ} \mathrm{C}$ then 35 cycles of $30 \mathrm{sec}$ at $94^{\circ} \mathrm{C}, 30 \mathrm{sec}$ at $60^{\circ} \mathrm{C}$ and $60 \mathrm{sec}$ at $72^{\circ} \mathrm{C}$, then $10 \mathrm{~min}$ at $72^{\circ} \mathrm{C}$. The amplified products were resolved by electrophoresis on an ethidium bromide-treated $2 \%$ agarose gel. Finally, the relative level of gene expression was expressed as the ratio of the target gene mean value to the geometric mean value of the reference gene, $\beta$-actin.

Antibodies and western blot analysis. Anti-phospho-PTEN (Ser380) rabbit polyclonal antibodies (pAb), anti-PTEN rabbit pAb, anti-phospho-AKT (Ser473) mouse monoclonal antibodies (mAb), anti-AKT rabbit pAb, anti-phospho-GSK-3 $\beta$ (Ser9) rabbit $\mathrm{pAb}$, anti-GSK-3 $\beta$ rabbit $\mathrm{pAb}$ were obtained from Cell Signaling Technology (Danvers, MA, USA). AntiGAPDH mouse mAb, anti- $\alpha$-tubulin mouse $\mathrm{mAb}$, horseradish peroxidase (HRP)-conjugated goat anti-mouse and anti-rabbit IgG were products of Santa Cruz Biotechnology (Santa Cruz, CA, USA). Western blot analysis was performed as previously described $(9,10)$.

Statistical analysis. All experiments were performed at least in triplicate. Numerical data were expressed as the means \pm SD. Two group comparisons were analyzed by the two-sided Student's t-test. P-values were calculated and a P-value of $<0.05$ was considered to indicate a statistically significant result.

\section{Results}

Progesterone attenuates cisplatin-induced inhibition of proliferation and cell cycle arrest in ovarian cancer cells. To determine the effect of progesterone on the cytotoxic effect of cisplatin, HO-8910 cells in DMEM with 5\% serum were exposed to $\mathrm{P} 4(1 \mu \mathrm{M})$, CDDP $(30 \mathrm{nM})$, or $\mathrm{P} 4(1 \mu \mathrm{M})$ plus CDDP (30 nM), respectively. MTT assay was then carried out to examine the effect of progesterone on cell proliferation. $\mathrm{P} 4$ alone did not have any effect on cell proliferation, and CDDP alone significantly inhibited the proliferation of HO-8910 cells. Notably, in the presence of $\mathrm{P} 4$, the inhibition of proliferation by CDDP was significantly attenuated (Fig. 1A). Consistent with these results, CDDP alone induced cell cycle arrest with a lower percentage $(15.90 \%)$ of cells in the S-phase when compared with these percentages in the cells exposed to $\mathrm{P} 4$ $(37.38 \%)$ or the control (39.96\%) (Fig. 1B). There was a higher fraction of P4 plus CDDP-treated cells in the S-phase (19.98\%) 
A

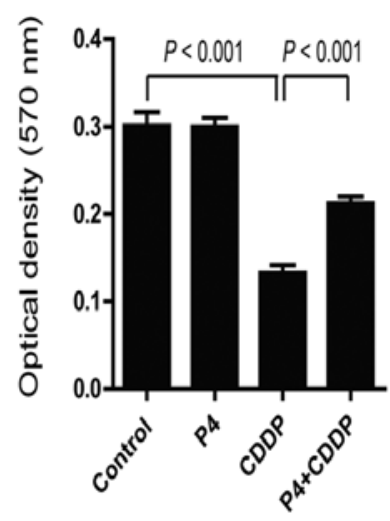

$\mathrm{B}$

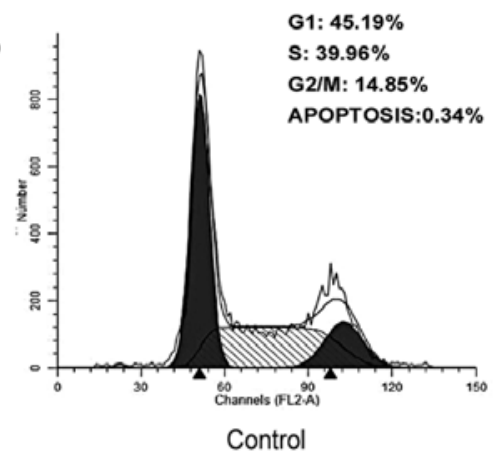

G1: $55.83 \%$

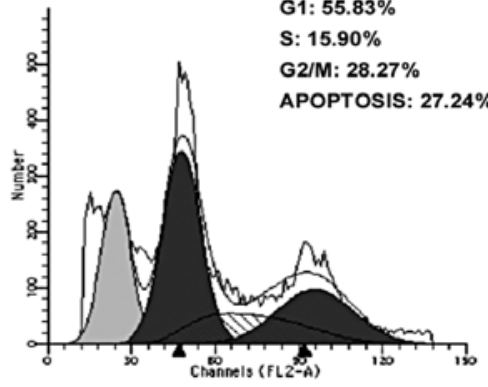

CDDP
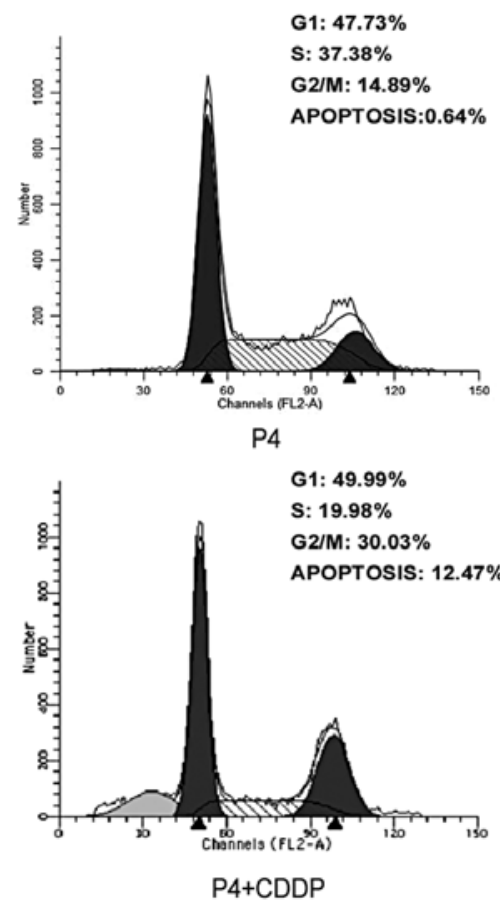

Figure 1. Progesterone attenuates cisplatin-induced inhibition of proliferation and cell cycle arrest in ovarian cancer HO-8910 cells. (A) MTT assay of the viability of HO-8910 cells treated with P4, CDDP or P4 plus CDDP, respectively. A representative column is shown from three independent experiments. (B) Flow cytometric analysis of the cell cycle distribution of the four groups. After serum starvation for $24 \mathrm{~h}$, HO-8910 cells were treated with P4, CDDP or P4 plus CDDP, respectively. Following incubation in complete medium for $16 \mathrm{~h}$, the cells were harvested and washed with PBS. The cell suspensions were treated and stained with propidium iodide followed by flow cytometric analysis of cell cycle distribution. A representative experiment is shown. The first peak corresponds to cells in the G0/G1 phase, the intermediary population to cells in the S-phase, and the second peak corresponds to cells in the G2/M-phase. Similar findings were observed in two repeated experiments.

than the fraction of cells treated with CDDP alone. Taken together, these data indicate that $\mathrm{P} 4$ attenuates the toxicity effect of CDDP in HO-8910 cells.

Capability of migration and invasion are partially restored by progesterone in cisplatin-treated HO-8910 cells. To determine the effect of $\mathrm{P} 4$ on migration and invasion of CDDPtreated HO-8910 cells, a wound healing assay and a Transwell chamber invasion assay were carried out. In the wound healing assay, CDDP significantly inhibited the migration of HO-8910 cells, while P4 attenuated the effect of CDDP and partially restored the migratory capability of the HO-8910 cells. After $24 \mathrm{~h}$ of treatment, the area of the wound closure of the CDDPtreated HO-8910 cells was decreased from $82.74 \pm 0.66 \%$ of the control to $10.25 \pm 2.17 \%$, while that of cells treated with P4 plus CDDP was increased to $24.28 \pm 3.94 \%$ compared with that of cells treated with CDDP alone (Fig. 2A). The same results were observed in the invasion assay. CDDP absolutely blocked the invasion of the HO-8910 cells from the upper to the lower chamber, however, $\mathrm{P} 4$ restored the action. After treatment of drugs for $18 \mathrm{~h}$, the number of invasive CDDP-treated HO-8910 cells on the lower chamber was $2 \pm 1$ per field, compared to the number of control or P4-treated cells which totaled $53 \pm 13$ or $51 \pm 11$ per field, respectively. Notably, the number of P4 plus CDDP-treated cells was increased to $17 \pm 6$ per field (Fig. 2B).

Multiple effect of progesterone on receptor expression profile in cisplatin-treated HO-8910 cells. Binding to receptors may be the initial step of progesterone to activate downstream signals and transcriptional factors. To determine the influence of P4 on the expression profile of PGR, PGRMC1 and PGRMC2, RT-PCR was carried out to detect the mRNA levels of these receptors in $\mathrm{HO}-8910$ cells exposed to $\mathrm{P} 4$ $(1 \mu \mathrm{M}), \mathrm{CDDP}(30 \mathrm{nM})$, or P4 $(1 \mu \mathrm{M})$ plus CDDP (30 nM), respectively. In the present study, the data showed a consistent result with that of Albrecht et al (11). Treatment with CDDP alone induced an increase in PGRMC1 mRNA by $~ 102.67 \%$ compared to the control, while PGRMC2 mRNA was reduced by $\sim 52.33 \%$. Meanwhile, a significant upregulation of mRNA transcription of the PGR by CDDP alone was also observed (Fig. 3). Notably, we first observed that P4 significantly inhibited the mRNA transcription of PGRMC1 and PGR in the CDDP-treated cells, whereas CDDP enhanced mRNA transcription of PGRMC2 (Fig. 3). It appeared that the expression profile of PGR/PGRMC1/2 must be tightly correlated with the anti-apoptotic effect of P4 on CDDP-resistance.

Progesterone upregulates AKT signaling in cisplatin-treated HO-8910 cells. PI3K/AKT signaling is a classical pathway which has been shown to be involved in many pathophysiological functions such as cell proliferation, migration, angiogenesis and tumorigenesis (12-14). Since we found that P4 rescued HO-8910 cells from CDDP-induced inhibitory effects, we inferred that PI3K/AKT signaling may be involved in this action. In the present study, immunoblot analysis showed that HO-8910 cells exposed to both P4 and CDDP (0.79-fold of activation normalized to the control) had a higher level of phos-AKT than the cells exposed to CDDP alone (0.37-fold of 

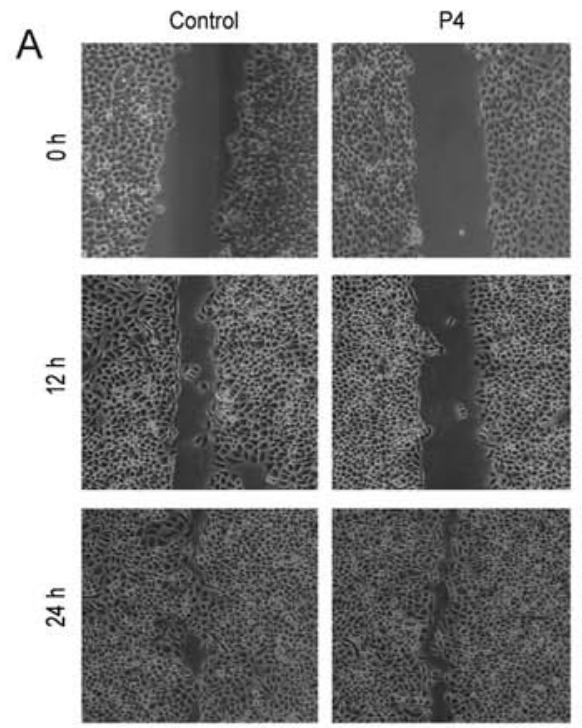

B

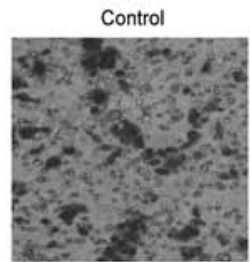

P4

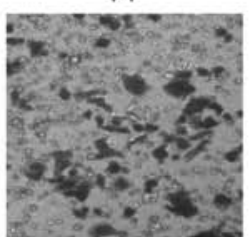

CDDP
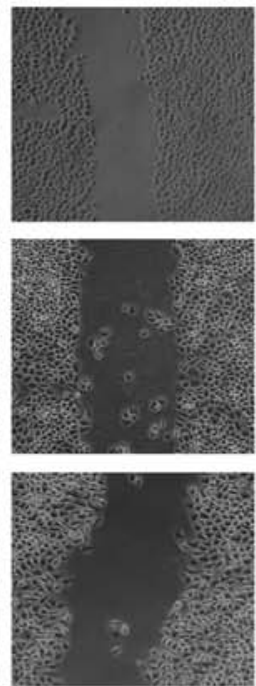

CDDP

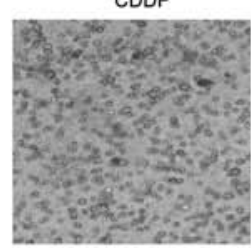

P4+CDDP
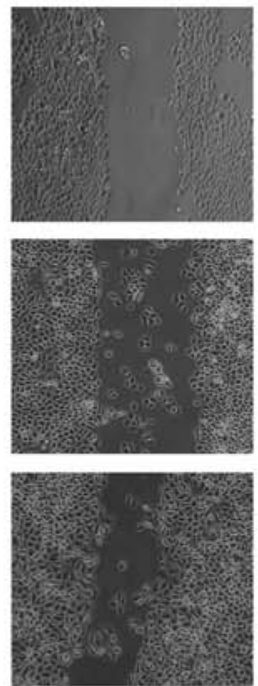

P4+CDDP

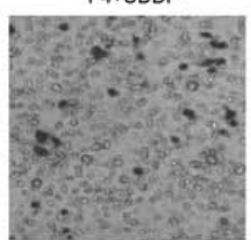

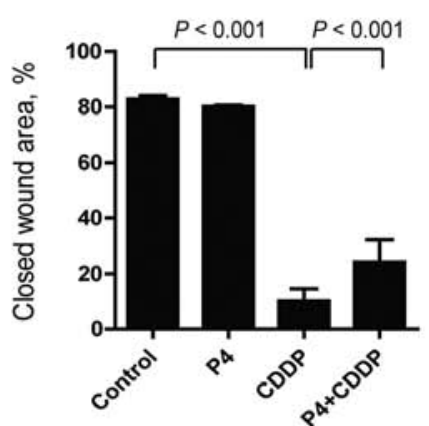

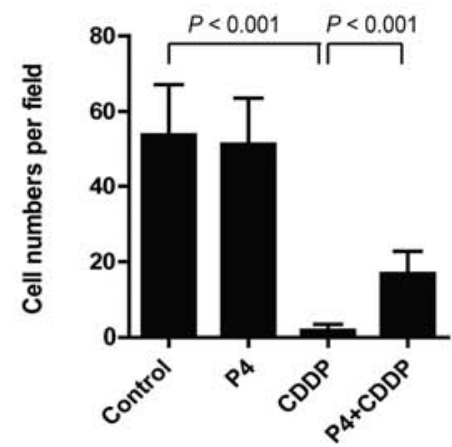

Figure 2. The migration and invasion abilities of cisplatin-treated HO-8910 cells were partially restored by progesterone. (A) A monolayer of HO-8910 cells was scratched with a sterile pipette tip. Repair of the lesion by cell migration without or with P4 or CDDP were photographed for the initial wounding $(0 \mathrm{~h})$ and at 12 and $24 \mathrm{~h}$ post-wounding. The areas of the lesions of the cells were quantified at the given times (left). A histogram at $24 \mathrm{~h}$ post-wounding is presented with the means \pm SE from three independent experiments (right). (B) Images of HO-8910 cells migrating through the porous membrane in the control, P4, CDDP and P4 plus CDDP groups (left). The average cell counts of invasive cells per 5 high powere fields (HPFs) in three groups (right). The data represent the results obtained from at least three independent experiments.

A

B
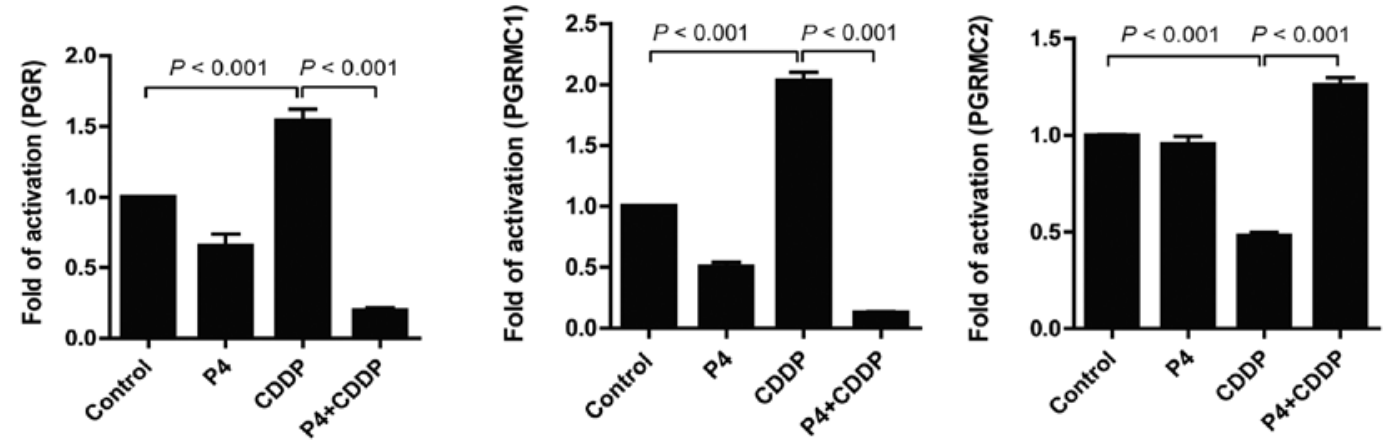

Figure 3. Multiple effect of progesterone on the expression profile of receptors in ciaplatin-treated HO-8910 cells. (A) Total RNA was extracted from HO-8910 cells pretreated with P4, CDDP or P4 plus CDDP for $24 \mathrm{~h}$, and RT-PCR with specific primers was carried out to detect the mRNA expression of PGR and PGRMC1/2. (B) Relative mRNA levels were normalized to that of $\beta$-actin by quantifying the optical density of the bands. The present data are consistent with another two independent experiments. 


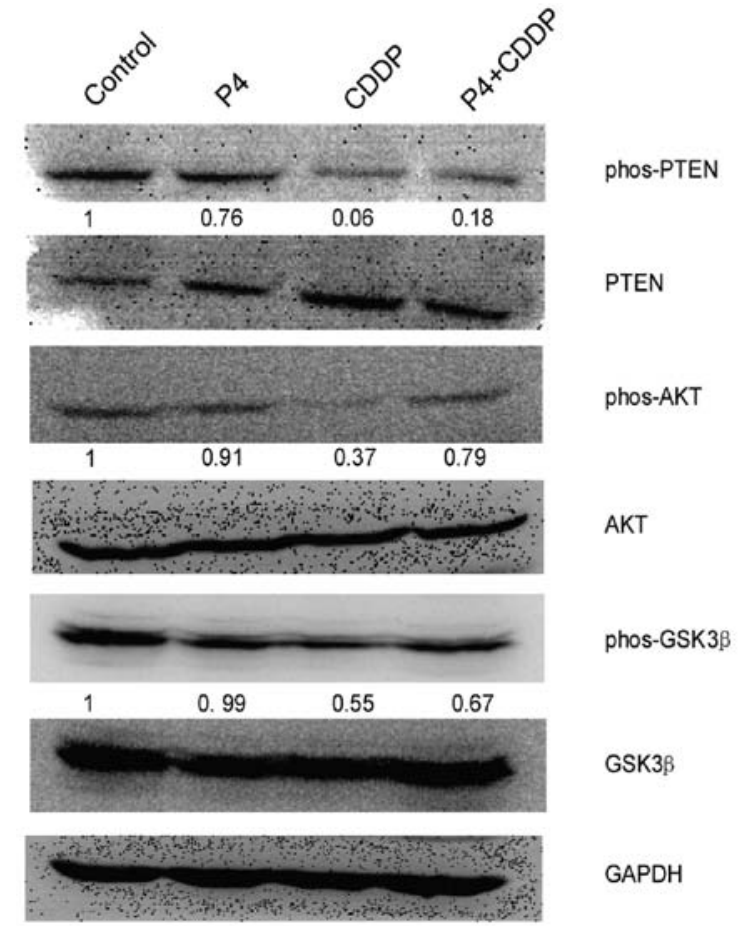

Figure 4. Progesterone upregulates AKT signaling in the cisplatin-treated HO-8910 cells. Phosphorylation of PI3K/PTEN/AKT/GSK-3 $\beta$ axis was detected by western blot analysis. HO-8910 cells treated with P4, CDDP or $\mathrm{P} 4$ plus CDDP for $48 \mathrm{~h}$ were lysed and subjected to the analysis.

activation). Meanwhile, higher phos-PTEN and phos-GSK-3 $\beta$ were also detected; the value of phos-PTEN was 0.18-0.06 and that of phos-GSK-3 $\beta$ was $0.67-0.55$, comparatively (Fig. 4). In the present study, we also found that $\mathrm{P} 4$ alone slightly downregulated the expression of phos-AKT, but the effect was not significant.

Inhibition of PI3K/AKT signaling blocks the anti-apoptotic effects of progesterone in $\mathrm{HO}-8910$ cells treated with cisplatin. To confirm the role of PI3K/AKT activation in the P4-mediated anti-apoptotic effects, LY294002, an inhibitor of PI3k/AKT signaling was added to the cell cultures before cell exposure to $\mathrm{P} 4(1 \mu \mathrm{M}), \mathrm{CDDP}(30 \mathrm{nM})$, or $\mathrm{P} 4(1 \mu \mathrm{M})$ plus CDDP (30 nM) as mentioned above. Inhibition of PI3K/AKT signaling by LY294002 significantly abrogated the anti-apoptotic effect of P4 in the CDDP-treated cells. Our data showed that HO-8910 cells treated with LY294002 had a high rate of apoptosis following addition of $\mathrm{P} 4(1 \mu \mathrm{M})$ plus CDDP $(30 \mathrm{nM})$ to the culture compared to that of the cells without LY294002 treatment (Fig. 5A). Consistent with this observation, HO-8910 cells pretreated with LY294002 and following P4 plus CDDP exposure restored the capability of migration (Fig. 5B). These striking findings confirmed a central role of AKT in the protective effect of $\mathrm{P} 4$ in the CDDP-resistant ovarian cancer HO-8910 cells.

\section{Discussion}

Ovarian cancer is the sixth most common cancer among women worldwide and is considered the leading cause of death from gynaecological malignancies in western countries $(1,15)$.
A

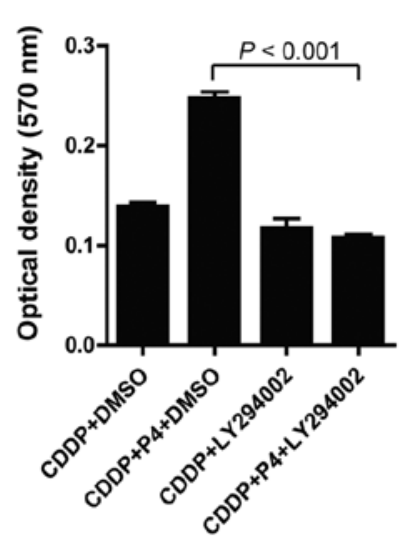

B
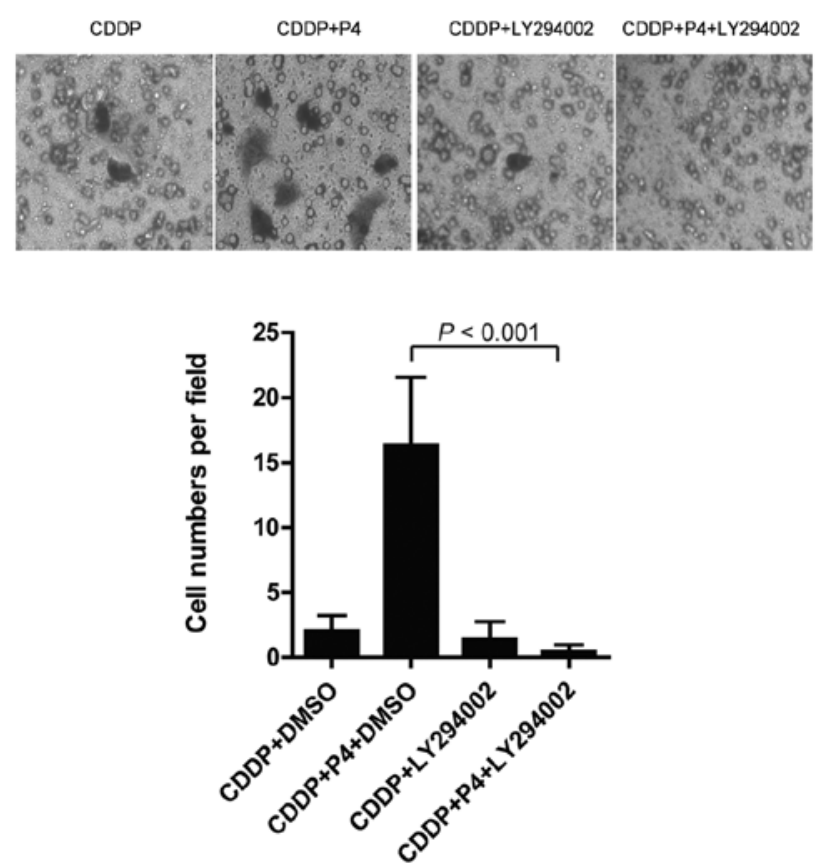

Figure 5. Inhibition of PI3K/AKT signaling blocks the restoration of cell viability and migration by progesterone in HO-8910 cells treated with cisplatin. (A) MTT assay of the viability of HO-8910 cells pretreated with CDDP or P4 plus CDDP following pretreatment with or without LY294002. (B) Migration of HO-8910 cells following treatment with CDDP or P4 plus CDDP and with pretreatment of LY294002 or without. Cells on the underside of the membrane were counted in a double-blind manner, from at least 5 high-power fields (HPFs) of three independent experiments.

The occurrence and development of cancer is a complicated process; pathogenic organisms, inflammatory factors, hormones followed by signal alterations are responsible (16-22). Regarding ovarian cancer as an endocrine neoplasia, dysfunction of the hormone system should be particularly noted. Until recently, many researchers have focused on the functions of $\mathrm{P} 4$ in ovarian cancer. Several authors have shown a suppressive effect of P4 on cancer development (23). However, the biologic effects of P4 correspond to the concentration of the drug, as described by Syed et al who reported that $\mathrm{P} 4$ at low concentrations exerts a growth promoting action while $\mathrm{P} 4$ at higher concentrations exerts a growth inhibitory effect (24-27). In the present study, we used a dose of $1 \mu \mathrm{M}$ P4 to detect its effect on CDDP-treated ovarian cancer cells. Our data revealed an anti-apoptotic effect of $\mathrm{P} 4$ with findings that $\mathrm{P} 4$ attenuated 
the toxic effect of CDDP and protected HO-8910 cells from CDDP-induced apoptosis in vitro. This finding is consistent with those reported by Peluso et al $(28,29)$. Importantly, as a hallmark of cancer malignancy, migration and invasion of HO-8910 cells were also restored by P4 following CDDP treatment.

To date, the mechanism by which $\mathrm{P} 4$ confer its anti-proliferative effect has been elucidated. The progesterone receptor (PGR) which has been proven relevant to the progression of ovarian cancer patients is responsible for the action (30-32). PGR is normally highly expressed on ovarian epithelial cells, while a decline in expression indicates a malignant alteration. Since P4 can mediate its transcriptional activity through a consensus glucocorticoid response element (GRE), which is not found in the PGRMC1 promoter but in PGR ones (33), the antiproliferative effect of $\mathrm{P} 4$ may be due in part to the antagonism of the binding of P4 to PGR. The present study first revealed a lower expression of PGR in the HO-8910 cell line in concordance with other ovarian cancer cell lines as previously described (34). Notably, CDDP increases the level of PGR as well as PGRMC1, while conversely decreases the level of PGRMC2. Most importantly, in CDDP-treated HO-8910 cells, $\mathrm{P} 4$ completely reversed the receptor expression profile by decreasing the level of PGR and PGRMC1, while increasing the level of PGRMC2. These results suggest the possibility that PGRMC2 instead of PGRMC1 may be crucial to the anti-apoptotic effect of P4 in CDDP-resistant HO-8910 cells. PGRMC1 and PGRMC2 both contain a cytochrome b5-like heme/steroid-binding domain and belong to the membrane-associated progesterone receptor (MAPR) family (35). Currently, PGRMC1 which is highly expressed in ovarian cancer and other cancer cells and was claimed to play an important role in chemotherapy resistance, has been extensively characterized in in vitro studies. Comparably little is known about PGRMC2. PGRMC2, sharing an amino acid identity of $\sim 89 \%$, is strongly homologous to PGRMC1, particularly regarding the intracellular portion containing the cytochrome b5-like domain (35). The present data contradict the notion that PGRMC1 plays an important role in CDDPresistance of P4 as reported by Peluso et al (29). Instead, our data support those of Albrecht et al (11) who reported that overexpression or knockdown of PGRMC1 had no effect on CDDP-induced apoptosis while overexpression of PGRMC2 induced significant CDDP resistance. Notably, a combination of $\mathrm{P} 4$ plus CDDP in the present study induced downregulation of PGRMC1 as well as PGR, while upregulating PGRMC2.

Given the high homology between PGRMC1 and 2, and the capability of both to interact with cytochrome P450 which has been shown to play a vital role in tumor progression and metastasis $(36,37)$, the pathophysiological effect of PGRMC1/2 on carcinogenesis should be further studied.

In addition to the alteration of the hormone microenvironment, signaling pathways may also play a crucial role in the process of carcinogenesis. PI3K/AKT is a classical signal transduction pathway which has been found to be involved in many types of cancers, including ovarian. The abnormal activation of PI3K/AKT may lead to the consequent onset, maintenance and chemo-resistance of ovarian cancer $(38,39)$. In the present study, significant activation of the PI3K/AKT pathway was detected in HO-8910 cells upon treatment with a combination of P4 and CDDP when compared with CDDP alone-treated cells. Notably, inhibition of the pathway by a chemical drug blocked the anti-apoptotic effect of P4 in CDDP-treated cells. Although, other signaling pathways may also play a role in the anti-apoptotic action of P4, PI3K/AKT signaling at least in part plays a role in this case.

Since PGRMC2 binds to cytochrome P450 proteins, 3A4 and $21 \mathrm{~A} 2$, while the former is associated with the activation of PI3K/AKT signaling in endothelial cells, the promising crosstalk between the P4/PGRMC1/2 pathway and PI3K/AKT signaling should be further investigated.

Taken together, $\mathrm{P} 4$ protects ovarian cancer cells from CDDP-induced apoptosis through activation of PI3K/ AKT survival signaling as well as by modulating the PGR/ PGRMC1/2 expression profile. Thus, the clinical combination of PI3K/AKT signaling inhibitors or a PGRMC antagonist with platinum-based chemotherapy may benefit the state and prognosis of ovarian cancer patients.

\section{Acknowledgements}

The present study was supported by the Department of Laboratory Medicine of Jiangsu Provincial Hospital of Traditional Chinese Medicine.

\section{References}

1. Salzberg M, Thurlimann B, Bonnefois $\mathrm{H}$, et al: Current concepts of treatment strategies in advanced or recurrent ovarian cancer. Oncology 68: 293-298, 2005.

2. O'Brien ME, Dowsett M, Fryatt I and Wiltshaw E: Steroid hormone profile in postmenopausal women with ovarian cancer. Eur J Cancer 30A: 442-445, 1994.

3. Halperin R, Hadas E, Langer R, Bukovsky I and Schneider D: Peritoneal fluid gonadotropins and ovarian hormones in patients with ovarian cancer. Int J Gynecol Cancer 9: 502-507, 1999.

4. Modugno F, Ness RB and Cottreau CM: Cigarette smoking and the risk of mucinous and nonmucinous epithelial ovarian cancer. Epidemiology 13: 467-471, 2002.

5. Franks AL, Lee NC, Kendrick JS, Rubin GL and Layde PM: Cigarette smoking and the risk of epithelial ovarian cancer. Am J Epidemiol 126: 112-117, 1987.

6. Green A, Purdie D, Bain C, Siskind V and Webb PM: Cigarette smoking and risk of epithelial ovarian cancer (Australia). Cancer Causes Control 12: 713-719, 2001.

7. Liu L, Wang J, Zhao L, et al: Progesterone increases rat neural progenitor cell cycle gene expression and proliferation via extracellularly regulated kinase and progesterone receptor membrane components 1 and 2. Endocrinology 150: 3186-3196, 2009.

8. Chen X, Cheng L, Jia X, et al: Human immunodeficiency virus type 1 Tat accelerates Kaposi sarcoma-associated herpesvirus Kaposin A-mediated tumorigenesis of transformed fibroblasts in vitro as well as in nude and immunocompetent mice. Neoplasia 11: 1272-1284, 2009

9. Tang Q, Qin D, Lv Z, et al: Herpes simplex virus type 2 triggers reactivation of Kaposi's sarcoma-associated herpesvirus from latency and collaborates with HIV-1 Tat. PLoS One 7: e31652, 2012.

10. Qin D, Feng N, Fan W, et al: Activation of PI3K/AKT and ERK MAPK signal pathways is required for the induction of lytic cycle replication of Kaposi's sarcoma-associated herpesvirus by herpes simplex virus type 1. BMC Microbiol 11: 240, 2011.

11. Albrecht $C$, Huck $V$, Wehling $M$ and Wendler $A$ : In vitro inhibition of SKOV-3 cell migration as a distinctive feature of progesterone receptor membrane component type 2 versus type 1 . Steroids 77 : 1543-1550, 2012.

12. Rodon J, Dienstmann R, Serra V and Tabernero J: Development of PI3K inhibitors: lessons learned from early clinical trials. Nat Rev Clin Oncol 10: 143-153, 2013. 
13. Wu J, Chen $\mathrm{C}$ and Zhao $\mathrm{KN}$ : Phosphatidylinositol 3-kinass signalings as a therapeutic target for cervical cancer. Curr Cancer Drug Targets 13: 143-156, 2013.

14. Polak R and Buitenhuis M: The PI3K/PKB signaling module as key regulator of hematopoiesis: implications for therapeutic strategies in leukemia. Blood 119: 911-923, 2012.

15. Sun CC, Ramirez PT and Bodurka DC: Quality of life for patients with epithelial ovarian cancer. Nat Clin Pract Oncol 4: 18-29, 2007.

16. Tangjitgamol S, Manusirivithaya S, Khunnarong J, Jesadapatarakul S and Tanwanich S: Expressions of estrogen and progesterone receptors in epithelial ovarian cancer: a clinicopathologic study. Int J Gynecol Cancer 19: 620-627, 2009.

17. Shanmughapriya S, Senthilkumar G, Vinodhini K, Das BC, Vasanthi $\mathrm{N}$ and Natarajaseenivasan K: Viral and bacterial aetiologies of epithelial ovarian cancer. Eur J Clin Microbiol Infect Dis 31: 2311-2317, 2012

18. Rosa MI, Silva GD, de Azedo Simoes PW, et al: The prevalence of human papillomavirus in ovarian cancer: a systematic review. Int J Gynecol Cancer 23: 437-441, 2013.

19. Gonzalez ER: Estrogen, progesterone receptors in ovarian cancer. JAMA 245: 1626, 1981.

20. Yin G, Chen R, Alvero AB, et al: TWISTing stemness, inflammation and proliferation of epithelial ovarian cancer cells through MIR199A2/214. Oncogene 29: 3545-3553, 2010.

21. Maccio A, Madeddu C, Massa D, et al: Hemoglobin levels correlate with interleukin- 6 levels in patients with advanced untreated epithelial ovarian cancer: role of inflammation in cancer-related anemia. Blood 106: 362-367, 2005.

22. Lee KB, Byun HJ, Park SH, Park CY, Lee SH and Rho SB: CYR61 controls p53 and NF- $\mathrm{BB}$ expression through PI3K/Akt/ mTOR pathways in carboplatin-induced ovarian cancer cells Cancer Lett 315: 86-95, 2012.

23. Murdoch WJ, Van Kirk EA, Isaak DD and Shen Y: Progesterone facilitates cisplatin toxicity in epithelial ovarian cancer cells and xenografts. Gynecol Oncol 110: 251-255, 2008.

24. Seeger H, Wallwiener D and Mueck AO: Is there a protective role of progestogens on the proliferation of human ovarian cancer cells in the presence of growth factors? Eur J Gynaecol Oncol 27: 139-141, 2006.

25. Ho SM: Estrogen, progesterone and epithelial ovarian cancer. Reprod Biol Endocrinol 1: 73, 2003.

26. Fauvet R, Dufournet Etienne C, Poncelet C, Bringuier AF, Feldmann G and Darai E: Effects of progesterone and antiprogestin (mifepristone) treatment on proliferation and apoptosis of the human ovarian cancer cell line, OVCAR-3. Oncol Rep 15: $743-748,2006$

27. Syed V, Ulinski G, Mok SC, Yiu GK and Ho SM: Expression of gonadotropin receptor and growth responses to key reproductive hormones in normal and malignant human ovarian surface epithelial cells. Cancer Res 61: 6768-6776, 2001
28. Peluso JJ, Gawkowska A, Liu X, Shioda T and Pru JK Progesterone receptor membrane component-1 regulates the development and cisplatin sensitivity of human ovarian tumors in athymic nude mice. Endocrinology 150: 4846-4854, 2009.

29. Peluso JJ: Progesterone signaling mediated through progesterone receptor membrane component- 1 in ovarian cells with special emphasis on ovarian cancer. Steroids 76: 903-909, 2011.

30. Lenhard M, Tereza L, Heublein S, et al: Steroid hormone receptor expression in ovarian cancer: progesterone receptor $\mathrm{B}$ as prognostic marker for patient survival. BMC Cancer 12: 553, 2012.

31. Leite DB, Junqueira MG, de Carvalho CV, et al: Progesterone receptor (PROGINS) polymorphism and the risk of ovarian cancer. Steroids 73: 676-680, 2008.

32. Gabra H, Watson JE, Taylor KJ, et al: Definition and refinement of a region of loss of heterozygosity at 11q23.3-q24.3 in epithelial ovarian cancer associated with poor prognosis. Cancer Res 56: 950-954, 1996.

33. Petz LN, Nardulli AM, Kim J, Horwitz KB, Freedman LP and Shapiro DJ: DNA bending is induced by binding of the glucocorticoid receptor DNA binding domain and progesterone receptors to their response element. J Steroid Biochem Mol Biol 60: 31-41, 1997.

34. Noguchi T, Kitawaki J, Tamura T, et al: Relationship between aromatase activity and steroid receptor levels in ovarian tumors from postmenopausal women. J Steroid Biochem Mol Biol 44: 657-660, 1993

35. Mifsud W and Bateman A: Membrane-bound progesterone receptors contain a cytochrome b5-like ligand-binding domain. Genome Biol 3: Research0068, 2002.

36. Szczesna-Skorupa E and Kemper B: Progesterone receptor membrane component 1 inhibits the activity of drug-metabolizing cytochromes $\mathrm{P} 450$ and binds to cytochrome $\mathrm{P} 450$ reductase. Mol Pharmacol 79: 340-350, 2011.

37. Oda S, Nakajima M, Toyoda Y, Fukami T and Yokoi T: Progesterone receptor membrane component 1 modulates human cytochrome p450 activities in an isoform-dependent manner. Drug Metab Dispos 39: 2057-2065, 2011.

38. Leake K, Singhal J, Nagaprashantha LD, Awasthi S and Singhal SS: RLIP76 regulates PI3K/Akt signaling and chemoradiotherapy resistance in pancreatic cancer. PLoS One 7: e34582, 2012

39. Kim TR, Cho EW, Paik SG and Kim IG: Hypoxia-induced SM22 $\alpha$ in A549 cells activates the IGF1R/PI3K/Akt pathway, conferring cellular resistance against chemo- and radiation therapy. FEBS Lett 586: 303-309, 2012. 